\title{
O ensino de Paleontologia nos anos finais do Ensino Fundamental: Fragilidades e potencialidades em livros didáticos de Ciências
}

The teaching of Paleontology in the final years of Elementary School: Weaknesses and potentialities in Science textbooks

\author{
La enseñanza de la Paleontología en los últimos años de la Escuela Primaria: Debilidades y \\ potencialidades en los libros de texto de Ciencias
}

Recebido: 30/05/2021 | Revisado: 09/06/2021 | Aceito: 13/06/2021 | Publicado: 27/06/2021

\author{
Bruno Peruzzi Peres \\ ORCID: https://orcid.org/0000-0002-3577-3631 \\ Universidade Federal do Pampa, Brasil \\ E-mail: brunossauro170897@gmail.com \\ Márcio da Mota Machado Filho \\ ORCID: https://orcid.org/0000-0002-3443-2931 \\ Universidade Federal do Pampa, Brasil \\ E-mail: marciotm95@gmail.com \\ Maurício Cendón do Nascimento Ávila \\ ORCID: https://orcid.org/0000-0002-6241-9697 \\ Universidade Federal do Pampa, Brasil \\ E-mail: mcn.avila@gmail.com \\ Edward Frederico Castro Pessano \\ ORCID: https://orcid.org/0000-0002-6322-6416 \\ Universidade Federal do Pampa, Brasil \\ E-mail: edwardpessano@unipampa.edu.br
}

\begin{abstract}
Resumo
A Paleontologia é o estudo da evolução da vida na Terra através dos registros deixados pelos organismos, explicando as mudanças e dispersão dos mesmos bem como as modificações do planeta ao longo de sua história geológica. Tendo em vista tal importância, um relevante aliado para o processo de disseminação deste conhecimento é o Livro Didático, material de caráter pedagógico que auxilia na abordagem de conhecimentos em aula de forma didática. Considerando o exposto, o objetivo deste trabalho foi investigar a abordagem da temática "paleontologia" em coleções de livros didáticos científicos dos anos finais do ensino fundamental. Este trabalho possui caráter quali-quantitativo, se tratando de uma pesquisa bibliográfica, que consistiu na análise de Livros Didáticos de ciências dos anos finais do ensino fundamental, do município de Uruguaiana, Rio Grande do Sul. Os resultados analisados denotam uma desvalorização da paleontologia a partir de uma abordagem superficial, incompleta ou ausente. Concluiu-se que se faz necessário a legitimação da temática paleontologia pelos Livros Didáticos em suas coleções, considerando a importância desta temática na articulação de diferentes conteúdos de ciências naturais, assim como entre as demais disciplinas curriculares.
\end{abstract}

Palavras-chave: Estudo dos fósseis; Período geológico; Livro de ciências.

\begin{abstract}
Paleontology is the study of the evolution of life on Earth through the records left by the organisms, explaining the changes and dispersion of them as well as the modifications of the planet throughout its geological history. In view of this importance, an importantally in the process of disseminating this knowledge is the Didactic Book, material of a pedagogical nature that helps in approaching knowledge in class in a didactic way. Considering the above, the objective of this work is to investigate the approach of the theme "paleontology" in collections of scientific textbooks from the final years of elementary school. This work has a qualitative and quantitative character in the case of bibliographic research, which consisted of the analysis of Didactic Books of science in the final years of elementary school, in the municipality of Uruguaiana, Rio Grande do Sul. The results analyzed denote a devaluation of paleontology in the materials due to the superficial, incomplete or absent approach to these issues. It was concluded that it is necessary to legitimize paleontology by Didactic Books in their collections, considering the importance of this theme in the articulation in different contents of natural sciences, as well as among the other curricular disciplines.
\end{abstract}

Keywords: Fossil study; Geological period; Sciense book. 


\begin{abstract}
Resumen
La paleontología es el estudio de la evolución de la vida en la Tierra a través de los registros que dejan los organismos, explicando sus cambios y dispersión, así como las modificaciones del planeta a lo largo de su historia geológica. Ante esta importancia, un aliado relevante para el proceso de difusión de este conocimiento es el Libro Didáctico, un material pedagógico que ayuda a abordar el conocimiento en clase de forma didáctica. Considerando lo anterior, el objetivo de este trabajo fue investigar el abordaje del tema "paleontología" en colecciones de libros de texto científicos de los últimos años de la escuela primaria. Este trabajo tiene un carácter cualitativo y cuantitativo, siendo una investigación bibliográfica, que consistió en el análisis de los libros de texto de ciencias de los últimos años de la escuela primaria, en el municipio de Uruguaiana, Rio Grande do Sul. Los resultados analizados muestran una devaluación de la paleontología basada en un abordaje superficial, incompleto o ausente. Se concluyó que es necesario legitimar el tema de la paleontología por los Libros Didácticos en sus colecciones, considerando la importancia de este tema en la articulación de diferentes contenidos de las ciencias naturales, así como entre las demás disciplinas curriculares.
\end{abstract}

Palabras clave: Estudio de fósiles; Período geológico; Libro de ciencia.

\title{
1. Introdução
}

No Brasil, desde 1929, os livros didáticos tem sido um dos principais recursos metodológicos e conceituais utilizados por professores(as) no ensino de ciências, subsidiando a construção de suas aulas, como também gerindo aporte teórico aos estudantes do ensino fundamental e médio (Brasil, 2017). Segundo Franco (1992), as considerações e preocupações com o uso dos livros didáticos iniciaram apenas em 1938, a partir da criação da Legislação do Livro Didático, instituída pelo Decreto-Lei 1006. Desde então, os livros já eram fiscalizados pelo Estado, por se tratarem de ferramentas ligadas à educação ideológica e/ou política além do ensino formal (Núñez et al., 2003).

Oliveira (2014) afirma que, desde a sua criação e implementação no meio educacional, os livros didáticos constroem sua história ligada diretamente ao histórico das próprias disciplinas escolares, o que faz todo sentido, considerando que, no ensino de ciências, tais materiais didáticos servem geralmente como um complemento ao conteúdo e abordagem do mesmo em aulas. Diversos estudiosos da área (Núñez et al., 2003; Romanatto, 2004; Frison et al., 2009) destacam a importância do Livro didático como referencial de orientação mais utilizado pelos professores e alunos para o processo de ensino e aprendizagem, auxiliando no desenvolvimento dos conteúdos, nos métodos avaliativos e até mesmo como um objeto de acesso cultural.

É importante deixar claro as principais funções deste referencial didático como "auxílio" e "orientação", pois cabe ao docente responsabilidade de seleção do material e conteúdo adequado, respeitando o contexto escolar na qual se legitima (Oliveira, 2014).

Como pontua Frison et al. (2009), a responsabilidade do(a) professor(a) perante os conhecimentos passados em aula é muito importante, pois ele não deve deixar de buscar informações em outras fontes de conhecimento por mais bem aceito e relevante que o LD seja. Um pensamento importante tendo em vista que a ciência está sempre em uma constante transformação, onde conhecimentos e fontes novas surgem a todo momento.

Sabemos que o desenvolvimento das novas tecnologias é algo presente na atualidade e que representa uma mudança significativa, inclusive no meio pedagógico através de novas mídias, textos digitais, entre outras ferramentas virtuais. Entretanto, mesmo com essas transformações metodológicas, muitos autores reforçam a ideia de que o livro didático ainda mantém a posição como mais fiel e completo aliado dentre todos os materiais utilizados nos meios escolares brasileiros (Delizoicov et al., 2002; Núñez et al., 2003; Frison et al., 2009; Oliveira, 2014). Segundo Cavalcante (2015), um dos motivos, se não o principal, da cultura de utilização do livro didático ainda ser claramente evidenciada, é de que as propostas contidas nos livros ainda serem as que melhor vão ao encontro do que preconizam os documentos oficiais da educação, mantendo-os como instrumento atual ideal na integração dos saberes.

A partir do exposto, entendemos que este recurso didático é um importante aliado do ensino, uma vez que diversos conteúdos das diferentes disciplinas curriculares exigem elementos visuais e conceituais que facilitem o aprendizado dos 
estudantes, como ilustrações, representações por imagens realistas e sugestões de dinâmicas, habitualmente dispostas nos LD.

Considerando o LD ser um excelente aliado no processo de ensino e aprendizagem, este fato se reforça no ensino de ciências pelo mesmo exigir um suporte metodológico conceitual por possuir maior complexidade em seus termos. Sendo necessário a presença de metodologias apresentadas em materiais didáticos para facilitar o entendimento dos estudantes. Como exemplo de conteúdo científico que se encaixa nesta complexidade terminológica está a paleontologia.

Segundo Sousa et al. (2016), a paleontologia é responsável por entendermos a evolução da vida na Terra através dos registros deixados pelo tempo e acaso. Descrição apropriada e que vai de encontro à etimologia da palavra pois palaios significa "antigo", antos representa o "ser" e logos o "estudo". As evidências que servem como base para o estudo são os fósseis, marcas deixadas por seres vivos do passado, como Almeida et al. (2013) descrevem detalhadamente:

O objeto de estudo da Paleontologia são os fósseis, que compreendem qualquer evidência, direta ou indireta de vida, com mais de 11.000 anos e incluem desde restos resistentes dos próprios organismos até vestígios ou marcas indicativas de atividades biológicas. Muitos seres encontrados atualmente têm representantes no registro fossilífero, demonstrando assim que nem todo grupo de determinado organismo fóssil está extinto (Almeida et al., 2013, p. 16).

Tais objetos comentados anteriormente são de suma importância não apenas para os paleontólogos, mas para a ciência como um todo. Segundo Martello et al. (2015) a paleontologia contribui relevantemente para muitos conhecimentos a serem ampliados, desde a evolução biológica até constituições geográficas e suas possíveis mudanças.

No Brasil, o estudo dos fósseis possui primeiros registros de pouco mais de 200 anos. Segundo relatos de naturalistas, estrangeiros já pesquisavam no país, expandindo seus conhecimentos de história e biologia, porém com pouca atenção recebida aos estudos e investimentos escassos para essa área. Tal situação só foi mudar a partir da fundação do Museu Nacional no Rio de Janeiro em 1818, quando comissões foram estabelecidas, assim como pesquisas na área foram estimuladas (Carvalho, 2010). Anelli (2002) corrobora destacando que desde então, a paleontologia no país tem favorecido várias áreas importantes do conhecimento.

Todo esse conteúdo acaba consequentemente tendo um destaque na área do ensino, onde autores como Schwanke \& Silva (2004), e Izaguirry et al. (2013) se referem à paleontologia como essencial, por auxiliar pessoas diretamente na compreensão de processos naturais operantes a bilhões de anos. Contudo, é notável a ausência de atenção para tal estudo por parte da escola, pois para maioria dos estudantes, tais conhecimentos são propagados pelas mídias (revistas, televisão, etc.) de forma distorcida e/ou confusa (Duarte et al., 2016).

De acordo com Schwanke e Silva (2004), nas escolas, é perceptível a importância de assuntos que compõe a biologia, a história e a geografia nos conteúdos escolares, entretanto, mesmo estando relacionada, a paleontologia ainda enfrenta dificuldades para se inserir nesse meio. Existem muitas possibilidades que justificam essas dificuldades, imposições em algumas vezes externas às próprias escolas. Para Vieira et al., (2010), Almeida et al. (2013) e Duarte et al. (2016), devido ao ensino das escolas terem um foco direcionado aos vestibulares, cursos preparatórios e cursos profissionalizantes, a atenção dos docentes é direcionada aos temas que são mais frequentes nos referidos exames, negligenciando alguns assuntos, dentre eles a paleontologia.

Os mesmos autores mencionam outro aspecto peculiar, que é a linguagem estritamente científica, sem o hábito de contextualizar, o que acaba por dificultar a associação e apropriação da temática pelos estudantes. Além disso, Almeida et al. (2013) menciona que podem haver deficiências conceituais em conteúdos dos livros didáticos direcionados ao ensino fundamental a respeito de paleontologia.

É importante ressaltar, que os Parâmetros Curriculares Nacionais - PCN, recomendam a paleontologia como temática científica no ensino básico brasileiro (Brasil, 1998). Mesmo sendo recomendado, segundo Almeida et al. (2013), os conteúdos 
relacionados à paleontologia são abordados geralmente de forma superficial, sem um tratamento legítimo e contextualizado, onde a temática acaba sendo negligenciada.

A partir da discussão exposta até o momento, o objetivo deste estudo buscou investigar a abordagem da temática "paleontologia" em coleções de livros didáticos de ciências dos anos finais do ensino fundamental, considerando a importância de sua articulação em diferentes conteúdos de ciências naturais, assim como entre as demais disciplinas curriculares. Autores como Carvalho (2010), Martello et al. (2015) e Sousa et al. (2016) afirmam com convicção que o estudo dos fosseis é uma área intrinsecamente interdisciplinar, existindo uma relação significativa com o ensino de ciências e compondo uma vasta diversidade de conceitos que podem ser abordados em aulas convencionais, como também em atividades extraclasse.

\section{Metodologia}

O presente estudo possui caráter quali-quantitativo, a partir de uma pesquisa bibliográfica que, segundo Gil (2019), consiste em um trabalho que se baseia em materiais com rigor científico previamente elaborados, cujas as fontes tenham natureza semelhante.

A pesquisa consistiu na análise de Livros Didáticos - LD de ciências dos anos finais do ensino fundamental, do município de Uruguaiana, RS. Foram procuradas a Secretaria Municipal de Educação e a $10^{\mathrm{a}}$ Coordenadoria Regional de Educação, onde foram solicitadas informações acerca das coleções mais selecionadas e utilizadas nas escolas da rede estadual e municipal na cidade de Uruguaiana em consonância com o Plano Nacional do Livro Didático - PNLD, de acordo com a base dedados disponível no site do Fundo Nacional de Desenvolvimento da Educação - FNDE.

Para constituição da amostra, foram selecionadas coleções de LD científicos de ciências da natureza dos anos finais do ensino fundamental, que por se tratar de uma disciplina que articula assuntos introdutórios à biologia, à química e à física, foram analisados em suas totalidades. É importante ressaltar que todas as coleções de livros avaliados já estão adaptadas à Base Nacional Comum Curricular - BNCC, documento normativo implementado em 2017, sendo parâmetro fundamental para a realização do planejamento curricular (Compiani, 2021). Os respectivos livros foram solicitados às mantenedoras e desenvolvida a análise conforme a matriz analítica. Os resultados obtidos foram avaliados com base nas diretrizes educacionais do Brasil, além de ter o apoio direto de estudos referentes as especificidades do conteúdo para devida comparação.

Para a análise, as coleções foram avaliadas a partir de uma matriz analítica adaptada de Castro et al. (2019), onde, para a mesma, foram desenvolvidas 6 categorias, somando 39 questões ao todo. Cada questão possui uma pontuação específica que resulta em um peso único para cada categoria, estabelecida conforme a relevância das temáticas a serem avaliadas apresentadas no Livro Didático. O peso de pontuação da categoria foi fundamentado a partir da importância que cada temática representa em relação aos assuntos que deveriam estar presentes ou ligados à Paleontologia.

É importante ressaltar que a análise foi feita por coleção, tendo em vista a divisão dos conteúdos presentes nas coleções serem a mesma para os 4 livros que as compõem. Para possibilitar que cada coleção fosse avaliada por completo, cada livro teve a análise de seu conteúdo utilizando a matriz adaptada e posteriormente somadas suas pontuações. A soma máxima da pontuação das categorias possui o peso aritmético de 100 pontos conforme ilustrado no Quadro 1. 
Quadro 1: Representação do valor da pontuação das categorias.

\begin{tabular}{|c|c|c|}
\hline CATEGORIA & PESO & NÚMERO DE QUESTÕES \\
\hline 1 - Geral & 10 & 5 \\
\hline 2 - Biológico & 31 & 3 \\
\hline 3 - Físico-química & 13 & 7 \\
\hline 4 - Geológica & 17 & 5 \\
\hline 5 - Sociocultural & 9 & 7 \\
\hline 6 - Didático & 20 & $\mathbf{3 9}$ \\
\hline Total & $\mathbf{1 0 0}$ & \\
\hline
\end{tabular}

Fonte: Adaptado de Castro et al (2019)

Ao final, os valores das categorias foram somados e constituíram a pontuação da coleção analisada e, conforme a pontuação final de cada coleção de livros, uma classificação era conceituada quando pontuados: de 0 a 24 como insuficiente (In), de 25 a 49 como básico (Ba), de 50 a 74 como regular (Re) e de 75 a 100 como ideal (Id).

\subsection{Categoria geral}

Categoria que analisa a totalidade de conteúdos que diz respeito à paleontologia, formada por 5 questões (apresentam uma ou mais opções) que juntas representam o peso de 10 pontos (Quadro 2). As demais categorias trabalham mais especificamente a abordagem dos temas que compõe de forma interdisciplinar a área paleontológica, representando $90 \%$ da pontuação final na matriz. Foi usado a CLDc2 de referência como parâmetro para estipular o ideal de número de páginas na questão 3, pois esta havia tido o melhor resultado neste caso.

Quadro 2: Questões da Categoria Geral e suas pontuações.

1) A obra apresenta o conceito de Paleontologia?

a) Suficiente (03 pontos), b) Regular (01 pontos), c) Insuficiente (0 pontos).

2) A obra apresenta, em conteúdos separados, conceitos que são abordados em Paleontologia (fossilização, eras geológicas, biologia evolutiva, etc)?

a) $\operatorname{Sim}(02$ pontos $)$, b) Não (0 pontos).

3) Número de páginas da Unidade dedicada aos conteúdos de Paleontologia (considerando as páginas destinadas ao conteúdo, figuras, textos, exercícios e leitura complementar):

a) Mais do que 20 (03 pontos), b) De 11 a 20 (02 pontos), c) De 1 a 10 (01 pontos), d) Nenhuma (0 pontos).

4) Quanto aos conteúdos da Paleontologia, a obra apresenta alguma mídia digital (CD, DVD, Cartão de memória, etc.) para ser usada no ambiente escolar?

a) $\operatorname{Sim}$ (01 pontos), b) Não (0 pontos).

5) São utilizados exemplos de fósseis brasileiros e regionais?

a) $\operatorname{Sim}$ (01 pontos), b) Não (0 pontos).

Fonte: Dados da Pesquisa. 


\subsection{Categoria biológica}

Categoria que caracteriza e avalia o conceito referente à biologia trabalhada em paleontologia, relacionando o que é apresentado nas coleções de LD com os temas que devem ser explorados. É formada por 12 questões representantes de 31 pontos da categoria (Quadro 3). Para as questões que investigam o número de espécies extintas citadas, a CLDc4 foi utilizada como base para estipular os valores a serem pontuados por possuir o maior número de espécies citadas, sendo mais de 4 .

Quadro 3: Questões da Categoria Biológica e suas pontuações.

\begin{tabular}{|c|}
\hline $\begin{array}{l}\text { 1) É apresentado e trabalhado o conceito de evolução? } \\
\text { a) Sim ( } 03 \text { pontos), b) Não ( } 0 \text { pontos). }\end{array}$ \\
\hline $\begin{array}{l}\text { 2) É apresentado algum modelo e/ou conceitualização de árvores filogenéticas? } \\
\text { a) Sim ( } 03 \text { pontos), b) Não (0 pontos). }\end{array}$ \\
\hline $\begin{array}{l}\text { 3) A obra menciona classificação taxonômica de espécies? } \\
\text { a) Sim ( } 03 \text { pontos), b) Não ( } 0 \text { pontos). }\end{array}$ \\
\hline $\begin{array}{l}\text { 4) Paleozoologia: Quantas espécies animais extintas são citadas? } \\
\text { a) Mais que } 4 \text { ( } 04 \text { pontos), b) de } 3 \text { a } 4 \text { ( } 02 \text { pontos), c) De } 01 \text { a } 02 \text { ( } 01 \text { pontos), d) nenhuma ( } 0 \text { pontos) }\end{array}$ \\
\hline $\begin{array}{l}\text { 5) Paleobotânica: Quantas espécies vegetais extintas são citadas? } \\
\text { a) Mais que } 4 \text { ( } 04 \text { pontos), b) de } 3 \text { a } 4 \text { ( } 02 \text { pontos), c) De } 01 \text { a } 02 \text { ( } 01 \text { pontos), d) nenhuma ( } 0 \text { pontos) }\end{array}$ \\
\hline $\begin{array}{l}\text { 6) As espécies são acompanhadas de: } \\
\text { a) Nome Científico ( } 01 \text { pontos), b) Nome Popular ( } 01 \text { pontos), c) Nome científico e popular ( } 02 \text { pontos). }\end{array}$ \\
\hline $\begin{array}{l}\text { 7) A obra relaciona achados paleontológicos com o estudo do ambiente? } \\
\text { a) Sim (02 pontos), b) Não ( } 0 \text { pontos). }\end{array}$ \\
\hline $\begin{array}{l}\text { 8) É indicado algum estudo comportamental provindo de achados paleontológicos? } \\
\text { a) Sim ( } 02 \text { pontos), b) Não ( } 0 \text { pontos). }\end{array}$ \\
\hline $\begin{array}{l}\text { 9) São mencionadas informações sobre icnofósseis (vestígios)? } \\
\text { a) } \operatorname{Sim}(02 \text { pontos }) \text {, b) Não (0 pontos). }\end{array}$ \\
\hline $\begin{array}{l}\text { 10) É trabalhado o conceito de anatomia comparada e sua relação com a evolução biológica (estruturas } \\
\text { análogas, homologas, vestigiais)? } \\
\text { a) Suficiente ( } 02 \text { pontos), b) Regular ( } 01 \text { pontos), c) Insuficiente ( } 0 \text { pontos). }\end{array}$ \\
\hline $\begin{array}{l}\text { 11) É trabalhado algum conceito voltado a padrões evolutivos entre espécies (coevolução, convergência } \\
\text { adaptativa, etc)? } \\
\text { a) Suficiente ( } 02 \text { pontos), b) Regular ( } 01 \text { pontos), c) Insuficiente ( } 0 \text { pontos). }\end{array}$ \\
\hline $\begin{array}{l}\text { 12) A obra se apresenta atualizada, utilizando nomes dos grupos taxonômicos abordados que seguem } \\
\text { agrupamentos lineanos" sem validade filogenética? } \\
\text { a) } \operatorname{Sim}(02 \text { pontos }) \text {, b) Não ( } 0 \text { pontos }) \text {. }\end{array}$ \\
\hline
\end{tabular}

Fonte: Dados da Pesquisa.

\subsection{Categoria físico-química}

Avalia a abordagem das coleções a respeito de assuntos paleontológicos compostos por eventos físicos e/ou químicos. Essa categoria é formada por 3 questões e tem peso de 13 pontos (Quadro 4). 
Quadro 4: Questões da Categoria Físico-Química e suas pontuações.

1) A obra cita em algum momento a utilização de métodos de datação absoluta (Carbono 14, por exemplo)?

a) Sim e relaciona com fósseis (05 pontos), b) Regular, apresenta sobre decaimento, mas não relaciona com fósseis (03 pontos), Não (0 pontos).

2) É trabalhado o processo de diagênese voltado à matéria biológica (fossilização)?

a) Suficiente (04 pontos), b) Regular (02 pontos), c) Insuficiente (0 pontos).

3) Número de páginas da Unidade dedicado a processos físico-químicos de unidades sedimentares (litificação, fossilização, etc):

a) Mais de 5 páginas (04 pontos), b) De 1 a 5 (02 pontos), c) Nenhuma (0 pontos).

Fonte: Dados da Pesquisa.

\subsection{Categoria geológica}

Busca analisar as caracterizações e abordagens de temas que envolvam assuntos e/ou matérias geológicos utilizados nos estudos paleontológicos. É composta por 7 questões com peso de 17 pontos.

Quadro 5: Questões da Categoria Geológica e suas pontuações.

1) Ao trabalharem rochas e seus tipos, são citadas rochas sedimentares e como se formam?

a) $\operatorname{Sim}(02$ pontos), b) Não (0 pontos).

2) A obra cita fatores geológicos apropriados ou não apropriados para fossilização?

a) Sim (02 pontos), b) Razoavelmente (01 pontos), c) Não (0 pontos).

3) O texto menciona o conceito de fóssil e quais são seus tipos?

a) Fósseis e seus tipos (03 pontos), b) Somente fósseis (01 pontos), c) Não (0 pontos).

4) É trabalhado o conceito de eras/períodos geológicos?

a) $\operatorname{Sim}(02$ pontos), b) Não (0 pontos).

5) A obra apresenta ilustrações demonstrando o conceito de tempo profundo?

a) $\operatorname{Sim}(02$ pontos), b) Não (0 pontos).

6) A obra aborda estratigrafia (estudo das camadas ou estratos que aparecem num corte geológico)?

a) $\operatorname{Sim}(03$ pontos), b) Não (0 pontos).

7) A obra trabalha a relação da estratigrafia com a idade das rochas ou fósseis encontrado nos estratos?

a) Sim (03 pontos), b) Não (0 pontos).

Fonte: Dados da Pesquisa.

\subsection{Categoria sociocultural}

Categoria formada por 6 questões com peso de 11 pontos, trata de assuntos que caracterizam a paleontologia na sociedade e sua importância cultural/econômica (Quadro 6). 
Quadro 6: Questões da Categoria Sociocultural e suas pontuações.

\begin{tabular}{l} 
1) A obra menciona ou relaciona a importância da Paleontologia para com a humanidade? \\
a) Sim (02 pontos), b) Não (0 pontos). \\
2) A obra menciona a Paleontologia em museus e/ou laboratórios (equipamentos, materiais e tecnologias)? \\
a) Sim (02 pontos), b) Não (0 pontos). \\
\hline 3) A obra chega a citar como a Paleontologia é apresentada na cultura pop (mídias audiovisuais, literatura, \\
etc) e seus equívocos? \\
a) Sim (01 pontos), b) Não (0 pontos). \\
4) A obra faz alguma ligação da Paleontologia com outras disciplinas (como história, geografia, etc)? \\
a) Sim ( 01 pontos), b) Não (0 pontos). \\
\hline 5) A obra menciona a importância econômica da Paleontologia relacionado a exploração de recursos \\
geológicos como carvão e petróleo? \\
a) Suficiente ( 03 pontos), b) Insuficiente ( 01 pontos), c) Não (0 pontos). \\
6) A obra traz um tópico com ideias e/ou curiosidades relacionadas a preservação de sítios, afloramentos \\
rochosos ou reservas usadas pelos paleontólogos em campo? \\
a) Sim ( 02 pontos), b) Regularmente ( 01 pontos), c) Não ( 0 pontos).
\end{tabular}

Fonte: Dados da Pesquisa.

\subsection{Categoria didática}

Aborda temas relacionados especificamente à construção didática dos livros para cada assunto que constitui a paleontologia, que além de contribuir para o estudo também auxilia na caracterização do mesmo. Formada por 6 questões em um peso equivalente à 18 pontos, conforme o Quadro 7, a seguir:

Quadro 7: Questões da Categoria Didática e suas pontuações.

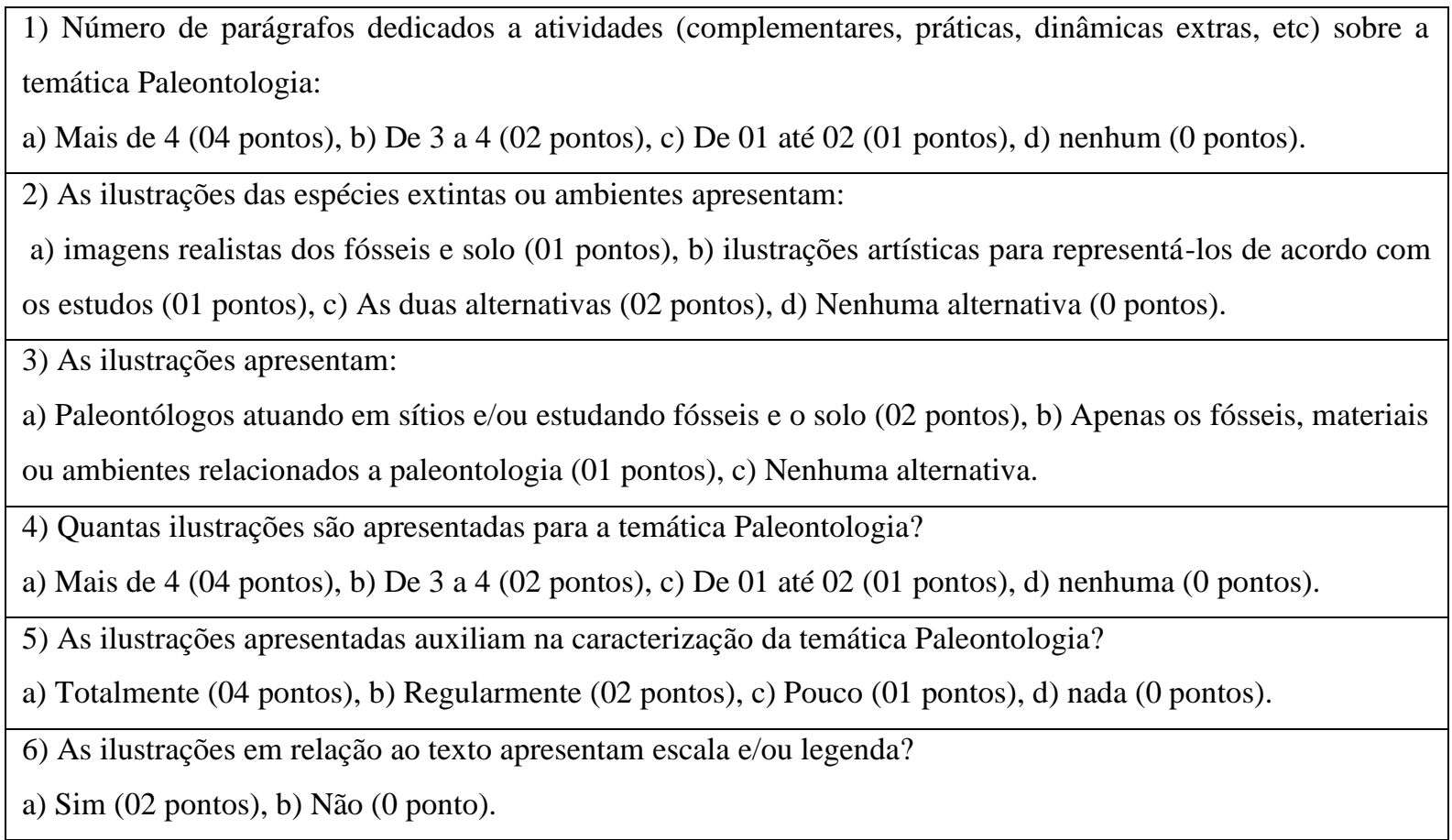

Fonte: Dados da Pesquisa. 
Por fim, foram somados os valores das categorias, constituindo a pontuação do livro analisado e enfim, da coleção. Conforme a pontuação final, cada coleção foi conceituada em determinada categoria. Após a aplicação da matriz, foi analisado em cada uma das Coleções de Livros Didáticos científicos - CLDc, o enfoque relativo a aspectos relevantes para paleontologia, descrevendo cada uma das obras.

\section{Resultados e Discussão}

Durante o levantamento, as informações registradas indicavam quais coleções de livros didáticos científicos foram mais selecionadas dentre todas as escolas da região, tomando nota de quais mais se repetiam para enfim estabelecer por soma o resultado. Foi dado destaque às 5 coleções mais utilizadas/requisitadas dentre as escolas públicas para o trabalho, ressaltando que a numeração segue a ordem de "ranking" sendo a CLDc1 a coleção que mais se repetiu dentre as informações de requerimento nas escolas, conforme mostra o Quadro 8:

Quadro 8: Coleções de Livros Didáticos Científicos - CLDc, selecionados para a pesquisa.

\begin{tabular}{|c|c|c|}
\hline Cod. & Título & Referência \\
\hline CLDc 1 & Projeto Araribá Mais - Ciências & Carnevalle (2018) \\
\hline CLDc 2 & Projeto Telaris: Ciências & Gewandsznajder \& Pacca (2018) \\
\hline CLDc 3 & Observatório de Ciências & Thompson \& Rios (2018) \\
\hline CLDc 4 & Ciências: Vida e Universo & Godoy (2018) \\
\hline CLDc 5 & Inspire Ciências & Bueno \& Macedo (2018) \\
\hline
\end{tabular}

Fonte: Elaborado a partir de dados obtidos junto à FNDE. Disponível em <www.fnde.gov.br> .

Todas as coleções se tratam de materiais atuais, e utilizados como fonte atualizada por conta de seus lançamentos, sendo as mesmas as últimas lançadas pelas editoras no ano de 2018. As coleções CLDc2 e CLDc3 são coleções que já estão em sua terceira edição, sendo que as outras coleções estão em sua primeira.

Por mais que a CLDc1 seja a mais utilizada pelas escolas da região, a CLDc2 possui uma organização bem mais clara dos conteúdos e textos explicativos. Todas as coleções seguem uma sequência de conteúdos e números de páginas semelhantes por livro, sempre contendo no mínimo uma ilustração por página, considerando um ponto positivo para o processo de aprendizagem, uma vez que imagens auxiliam a visualização e compreensão do contexto estudado. Neste quesito, a CLDc2 merece destaque, contendo ilustrações mais detalhadas e com descrições das mesmas mais completas. De maneira geral, a CLDc4 e a CLDc5 são as que apresentam menores quantidades de conteúdo relacionado ao tema Paleontologia.

\subsection{Pontuação das coleções por categoria}

Após a análise completa de todos os 20 livros das 5 coleções selecionadas para este trabalho, ressaltando serem 4 livros por coleção, foi distribuída a pontuação relativa a cada questão. Foi possível observar características interessantes, como o fato de nenhum dos LD do $8^{\circ}$ ano das coleções apresentar uma temática que tivesse alguma ligação com paleontologia, além de definições incompletas e/ou superficiais que serão discutidas mais a seguir.

\subsubsection{Categoria Geral}

Pela categoria Geral, as análises mostraram que nenhuma coleção obteve pontuação máxima nesta categoria, sendo que CLDc2 foi a que chegou mais perto com pontuação 7. Um dos pontos negativos observados em todas as coleções é a 
superficialidade na definição de paleontologia, resultando em pontuações baixas como no caso de CLDc3 e CLDc5, sendo as mais baixas, evidenciadas no Quadro 9.

Quadro 9: Pontuação de cada coleção analisada referente à Categoria Geral, onde 10 representa a pontuação máxima.

\begin{tabular}{|c|c|c|c|c|c|c|}
\hline COLEÇõES & Q1 & Q2 & Q3 & Q4 & Q5 & Total \\
\hline CLDc1 & 1 & 2 & 3 & 0 & 0 & $\mathbf{6}$ \\
\hline CLDc2 & 1 & 2 & 3 & 0 & 1 & $\mathbf{7}$ \\
\hline CLDc3 & 0 & 2 & 1 & 0 & 0 & $\mathbf{3}$ \\
\hline CLDc4 & 1 & 2 & 2 & 0 & 0 & $\mathbf{5}$ \\
\hline CLDc5 & 1 & 2 & 1 & 0 & 0 & $\mathbf{4}$ \\
\hline
\end{tabular}

Fonte: Dados da Pesquisa.

É preocupante observar a superficialidade das descrições sobre paleontologia nos LD, sendo que muitos autores, como Zucon et al. (2010), Martello et al. (2015) e Sousa et al. (2016), destacam a relevância na promoção do conhecimento paleontológico. Segundo Zucon et al, (2010, p. 2), "Dentre as áreas das Ciências Naturais, a Paleontologia é aquela que vem apresentando um desenvolvimento promissor nas últimas décadas, ocupando local de destaque na busca pela compreensão da evolução dos seres vivos e da história da Terra".

Portanto, a compreensão de processos naturais dos seres vivos e da própria Terra merecem seu espaço no meio científico e destaque para o âmbito educacional. Para Novais et al. (2015) a forte influência da ciência no mundo moderno é clara, portanto, se faz necessário que as fontes científicas, principalmente os LD, que são referenciais conceituais e didáticos na educação básica, se mantenham atualizados e completos para que os conhecimentos tenham maior relevância para a aprendizagem dos estudantes. Concordamos, a partir desta afirmação com a necessidade de a temática "Paleontologia" possuir sua legitimidade, a partir de informações científicas adequadas para cada nível de ensino em LD, assim como instruções didáticas que promovam a sua contextualização no ensino de ciências.

Existem artifícios/meios que auxiliam a didática para com o ensino da paleontologia, um exemplo comum está nas ilustrações. Segundo Núñez et al. (2003), a presença de figuras para o ensino de ciências é recomendada em materiais didáticos para facilitar a compreensão dos conteúdos, dando maior contexto aos mesmos. Como pondera Ghilardi (2007), na paleontologia são fundamentais traduções visuais apropriadas, e complementares às informações, para melhor entendimento da mesma.

Como complemento ao ensino de ciências, também existem mídias digitais, que cada vez mais estão tomando seu espaço no meio das ferramentas pedagógicas. $\mathrm{O}$ que se faz relevante mencionar que nenhuma das coleções trazia consigo nenhum material digital extra ou menção à atividade digital. Resultado curioso, pois o uso de instrumentos digitais como recurso para o processo de ensino e aprendizagem é um excelente facilitador da aprendizagem, aprimorando a didática do professor em meio às aulas, com a tendência de serem recursos muito mais atrativos para os próprios estudantes (Jordão, 2010).

Podemos considerar os referidos recursos como facilitadores didáticos, permitindo que os conteúdos escolares não se apresentem de forma isolada, fragmentada ou distantes de seus contextos distintos. A contextualização no ensino é, segundo Kato e Kawasaki (2011) a proposta de situar e relacionar o conhecimento que é dado nas escolas, aos seus vários contextos de produção, apropriação e utilização. Para Carvalho (2010), na paleontologia não é diferente, pois ela integra muitas áreas do conhecimento científico para melhor relação no cotidiano. Portanto, o despertar do interesse dos estudantes resulta em uma 
construção mais efetiva de conhecimentos paleontológicos mais contextualizados, relacionando melhor a história biológica e geológica da Terra com o próprio ambiente habitual, situação que pode ser reforçada com o uso de ferramentas lúdicas.

Quanto às menções a fósseis brasileiros, a CLDc2 foi a única a trazer algum exemplo a respeito, sendo um fóssil de um peixe encontrado na região do Ceará, sem informações científicas a mais (p. 23). Fato que representa uma possível deficiência nos LD em negligenciar a exploração de um diversificado acervo de materiais paleontológicos disponíveis, pois, como afirma Sousa et al. (2016), o território nacional é um grande potencial na área de paleontologia por possuir fósseis no mínimo singulares comparado ao resto do mundo.

A definição da temática e suas vertentes em poucas palavras e a abordagem breve já sugere um possível esquecimento da mesma nas coleções analisadas. Representando uma falta de reconhecimento das coleções a respeito dos conhecimentos paleontológicos tendo em vista sua importância e potencial no ensino de ciências.

\subsubsection{Categoria Biológica}

Foi possível observar que todas as coleções apresentam conceitos de termos evolucionistas (evolução, filogenia, taxonomia, etc.), entretanto, nem todas se aprofundam em áreas mais especificas como tipos de fósseis e/ou paleobotânica, a exemplo da CLDc2 e CLDc5. As CLDc1 e CLDc4 foram as únicas a apresentar no mínimo uma espécie vegetal extinta, como também foram as únicas a mencionar os "icnofósseis". Sobre temáticas evolucionistas, a CLDc3 é a que melhor define com clareza os conceitos, dispondo de informações mais condizentes com a atualidade, mantendo-se como uma das maiores pontuações dentre as coleções como observado no Quadro 10.

Quadro 10: Pontuação de cada coleção analisada referente à Categoria Biológica, onde 31 representa a pontuação máxima.

\begin{tabular}{|c|c|c|c|c|c|c|c|c|c|c|c|c|c|}
\hline COLEÇõES & Q1 & Q2 & Q3 & Q4 & Q5 & Q6 & Q7 & Q8 & Q9 & Q10 & Q11 & Q12 & Total \\
\hline CLDc1 & 3 & 3 & 3 & 2 & 1 & 1 & 2 & 0 & 2 & 2 & 1 & 0 & $\mathbf{2 0}$ \\
\hline CLDc2 & 3 & 3 & 3 & 4 & 0 & 2 & 2 & 2 & 0 & 0 & 0 & 0 & $\mathbf{1 9}$ \\
\hline CLDc3 & 3 & 3 & 3 & 2 & 0 & 2 & 2 & 0 & 0 & 2 & 2 & 2 & $\mathbf{2 1}$ \\
\hline CLDc4 & 3 & 3 & 3 & 4 & 1 & 2 & 2 & 2 & 2 & 2 & 0 & 0 & $\mathbf{2 4}$ \\
\hline CLDc5 & 3 & 3 & 0 & 2 & 0 & 1 & 2 & 0 & 0 & 1 & 0 & 0 & $\mathbf{1 2}$ \\
\hline
\end{tabular}

Fonte: Autores.

Em relação a biologia evolutiva, os conteúdos apresentados pelas coleções estão bem estruturados e relativamente completos quando analisados no geral, algo positivo de se esperar tendo em vista sua importância para a compreensão da origem dos seres vivos na Terra. Para Araújo (2017), a evolução é o estudo que remete às mudanças existentes na vida ao redor do tempo, que se faz muito importante no ensino por se tratar da única explicação cientifica para a origem e diversidade da vida.

Na paleontologia, utiliza-se muito desse conceito para a estruturação de seus estudos, tanto por se tratar de conhecimentos que derivam dos fatos históricos, quanto pela biologia que é trabalhada como uma vertente básica nessa temática. Segundo Zamberlan e Silva (2009), a evolução, dentre suas teorias, deve seguir esse pensamento no ensino, de que se trata de um princípio organizador dos conhecimentos biológicos como um todo.

Outro tema relacionado a evolução, que possui uma expressiva abordagem nas coleções, é a sistemática filogenética para classificação de espécies e temas relacionados. Quando é promovido o ensino sobre a dispersão da vida e seu desenvolvimento evolutivo em materiais didáticos, é importante e necessário que sejam fornecidos conceitos que auxiliem o 
estudante a compreender e visualizar de forma clara as origens remotas e ancestralidade conforme ocorre na abordagem sobre classificação taxonômica e a filogenia.

A ausência da taxonomia na CLDc5 é preocupante, por mais que contenha um exemplo de árvore filogenética, afinal de contas, mesmo com tais modelos que auxiliam a ciência na sustentação e visualização de grupos naturais, são os agrupamentos taxonômicos modernos que nos permitem identificar o real parentesco dentre os organismos vivos e não apenas por similaridade (Neto \& Paesi, 2017).

Todos esses assuntos são elementos essenciais na área biológica da paleontologia, tema este que, sendo trabalhado em escolas, se torna uma ferramenta fundamental na compreensão da biologia evolutiva, até mesmo ecologia e distribuição de seres vivos atuais (Dos Reis et al., 2005). De acordo com Novais et al. (2015), a paleontologia é um tema essencial em discussões sobre os seres do passado, reforçando a associação de espécies extintas com os grupos viventes.

Dentre todos as questões relacionadas a identificações taxonômicas, quando buscado nas coleções a respeito de nomenclatura científica e popular, apenas as CLDc2, CLDc3 e CLDc4 foram satisfatórias em apresentar ambos registros em suas ilustrações/figuras de espécies extintas. A partir do momento que LD indicam tais detalhes, aprender a respeito da biodiversidade já se torna mais acessível, principalmente por reforçar o contato filogenético entre espécies (Neto \& Paesi, 2017). Para a ciência, inclusive a paleontologia, a nomenclatura científica de espécies indica a proximidade entre organismos, incluindo seus parentes extintos, sendo aos pesquisadores uma base para comunicação entre estudos acadêmicos (Leite \& De Sá, 2010).

Elementos como biologia evolutiva, taxonomia e sistemática filogenética se mantêm presentes e aprofundados, mesmo que um pouco desatualizados. Alguns dos assuntos biológicos não apresentavam falta de definição quando o foco é paleontologia, porém tratados de forma supervalorizada, como resumir a temática a fósseis e/ou representações de dinossauros para citação de espécies extintas, promovendo tais assuntos de forma excessiva em detrimento a outros que compõe a temática.

\subsubsection{Categoria Físico-química}

Foi possível diagnosticar que são raros os conceitos mais completos referentes aos processos físico-químicos trabalhados na paleontologia que apareceram nas coleções. A CLDc2, sendo possível observar que é a coleção com a maior pontuação, é a única que apresenta informações sobre decaimento radioativo, mas não relacionou diretamente com datação absoluta. Com 2 pontos totais, a CLDc1 não estimula nem o básico sobre processos de fossilização da matéria biológica, conforme observado no Quadro 11.

Quadro 11: Pontuação de cada coleção analisada referente à Categoria Físico-química, sendo 13 é a pontuação máxima.

\begin{tabular}{|c|c|c|c|c|}
\hline COLEÇõES & Q1 & Q2 & Q3 & Total \\
\hline CLDc1 & 0 & 0 & 2 & $\mathbf{2}$ \\
\hline CLDc2 & 3 & 4 & 2 & $\mathbf{9}$ \\
\hline CLDc3 & 0 & 2 & 2 & $\mathbf{4}$ \\
\hline CLDc4 & 0 & 4 & 2 & $\mathbf{4}$ \\
\hline CLDc5 & 0 & 2 & 2 & $\mathbf{6}$ \\
\hline
\end{tabular}

Fonte: Dados da Pesquisa.

Nesta categoria, são mais escassas as ocorrências relacionadas à temática paleontologia nos LD observados. Em LD de ciências no geral, os conteúdos de química e física costumam focar em estruturas atômicas e leis, desfavorecendo sua articulação para discussões com paleontologia (Sousa et al., 2016). 
Conteúdos como datação absoluta por carbono, apenas foram evidenciados na CLDc2, contudo, de forma superficial, reforçando a negligência dos materiais para com os processos físico-químicos na paleontologia. O decaimento radioativo é, segundo Sousa et al. (2016), muito importante para o estudo da datação dos fósseis e, portanto, algo que deveria ser melhor conduzido no ensino para discussões de viés paleontológico.

Um tema considerado essencial e que deve ocorrer em todos os LD é a fossilização, pois segundo Zucon et al. (2010), trata-se de um assunto muito importante para o ensino de paleontologia, sendo resultado exatamente de processos físico e químicos que atuam no ambiente. Tal importância é abordada pela maioria das coleções analisadas, com exceção da CLDc1, que acaba não deixando clara a presença de transformações físico-químicas na natureza aos estudantes.

Com isso, é observado na análise que no geral as coleções não trazem uma abordagem envolvente a respeito de processos físicos ou químicos a respeito de paleontologia. Pecando em fatores importantes para a facilitação do ensino da temática e compreensão de processos naturais.

\subsubsection{Categoria Geológica}

Apesar de todas coleções apresentarem informações sobre rochas, principalmente sedimentares, pouco ou nada se trabalha a respeito de fatores geológicos para a formação dos fósseis, como no caso da CLDc4. É reforçado de forma visualmente detalhada as mudanças geológicas do planeta, conforme o tempo, nas coleções CLDc1, CLDc2 e CLDc5; sendo de forma mais superficial, na CLDc4; e ausente, na CLDc3 (Quadro 12).

Quadro 12: Pontuação de cada coleção analisada referente à Categoria Geológica, onde 17 representa a pontuação máxima.

\begin{tabular}{|c|c|c|c|c|c|c|c|c|}
\hline COLEÇÕES & Q1 & Q2 & Q3 & Q4 & Q5 & Q6 & Q7 & Total \\
\hline CLDc1 & 2 & 1 & 3 & 0 & 2 & 3 & 0 & $\mathbf{1 1}$ \\
\hline CLDc2 & 2 & 1 & 1 & 2 & 2 & 3 & 3 & $\mathbf{1 4}$ \\
\hline CLDc3 & 2 & 1 & 1 & 0 & 0 & 3 & 0 & $\mathbf{7}$ \\
\hline CLDc4 & 2 & 0 & 3 & 0 & 1 & 0 & 3 & $\mathbf{9}$ \\
\hline CLDc5 & 2 & 1 & 3 & 0 & 2 & 0 & 3 & $\mathbf{1 1}$ \\
\hline
\end{tabular}

Fonte: Dados da Pesquisa.

No que se refere a geologia, o estudo das rochas tem um papel fundamental na ciência, sendo uma área que classifica a composição mineral do nosso solo e mapeia regiões inteiras através de atributos geológicos que se diferem por meio de cor, textura, idade e outros atributos (Carneiro et al., 2005). É observado que esse assunto não foi aprofundado nas coleções, desprezando sua importância, já que tais mapeamentos, denominados "Formações", tendem a ser úteis ao trabalho de paleontólogos, principalmente em ambientes deposicionais, que costumam ser formados pelo acúmulo de materiais sedimentares (Zucon et al., 2010).

Na paleontologia, fatores geológicos são um dos cernes do estudo, principalmente pelo fato de seu objeto principal ser o Fóssil, registro geológico deixado por seres vivos do passado (Almeida et al., 2013). Esse registro está depositado frequentemente no solo, e, segundo Rohn (2000), para seu maior entendimento e classificação, como a idade do fóssil e até a respeito do ambiente, é preciso aplicar conceitos da Estratigrafia. $\mathrm{O}$ autor a define como estudo científico dos estratos sedimentares, ciência essa que traz à paleontologia informações importantes como a ordenação cronológica de minerais, fósseis e até mesmo eventos geológicos. Informações cruciais para o entendimento do passado terrestre e que não deveriam ser deixadas de lado em LD, onde neste trabalho, por mais superficial, em nenhuma coleção esse tema se mostrou ausente. 
A relação dos estratos com o tempo e as eras geológicas na CLDc3 e CLDc4 se mostraram ineficientes. A CLDc2 passou as informações de forma mais ideal dentre as coleções, demonstrando de forma completa e didática as ocasiões de mudanças da biosfera e litosfera. Temas que Carneiro et al. (2005) se refere como momentos importantes na história geológica da Terra, ressaltando que, através de descontinuidades estratigráficas entre camadas no solo, é possível entender o surgimento de grupos de organismos, assinalando momentos relevantes na evolução biológica.

Além de surgimentos, também se estuda a ausência na continuidade de organismos, que seguindo padrões, mostra à ciência a possibilidade de diversas extinções, tanto específicas quanto em massa, onde constroem divisões do percurso da vida ao redor do tempo, uma das maiores importância no estudo do tempo geológico (Carneiro et al., 2005).

Questões como eras geológicas, extinções em massa e datação absoluta, que estão diretamente relacionadas à Paleontologia e que são multifacetadas, exigindo maior conhecimento articulado entre áreas das ciências da natureza, são menos valorizadas e promovidas nas coleções analisadas. Tais resultados deixam clara a limitação do conteúdo a disciplinas pontuais e específicas.

\subsubsection{Categoria Sociocultural}

Foi possível identificar que todas coleções apresentam alguma ligação da paleontologia com pelo menos uma outra área do conhecimento (geografia na maioria dos casos), citando a importância da mesma com a humanidade, mesmo que de maneira breve. Porém, nada relacionado à cultura pop, literatura, museus, equipamentos laboratoriais, tecnologia usada por paleontólogos e preservação de sítios de escavação são citados nas coleções em questão (Quadro 13).

Quadro 13: Pontuação de cada coleção analisada referente à Categoria Sociocultural, onde 9 representa a pontuação máxima.

\begin{tabular}{|c|c|c|c|c|c|c|c|}
\hline COLEÇÕES & Q1 & Q2 & Q2 & Q4 & Q5 & Q6 & Total \\
\hline CLDc1 & 2 & 0 & 0 & 1 & 1 & 0 & $\mathbf{4}$ \\
\hline CLDc2 & 2 & 0 & 0 & 1 & 0 & 0 & $\mathbf{3}$ \\
\hline CLDc3 & 2 & 0 & 0 & 1 & 1 & 0 & $\mathbf{4}$ \\
\hline CLDc4 & 2 & 0 & 0 & 1 & 3 & 0 & $\mathbf{6}$ \\
\hline CLDc5 & 2 & 0 & 0 & 1 & 0 & 0 & $\mathbf{3}$ \\
\hline
\end{tabular}

Fonte: Dados da Pesquisa.

A paleontologia age em vários campos da ciência, sendo responsável por expor a história do planeta e dos seres que aqui viveram através das épocas históricas. Segundo Manzig (2015), o estudo paleontológico tem grande valor para a humanidade na criação de uma consciência a respeito de valores culturais e preservação de patrimônio natural.

Carvalho (2010) destaca a importância de a paleontologia ser discutida em outras disciplinas escolares que possuem temas que a compõem, como no caso da geografia, a história e até mesmo a matemática, proporcionando uma aprendizagem mais significativa e multidisciplinar. Por conta disso, inserir tais conhecimentos se torna fundamental para a sociedade, tanto pelo ensino quanto por outros meios informais de aprendizagem.

Nas escolas, segundo Almeida et al. (2013), o que mais auxiliaria o alcance dos conhecimentos paleontológicos seriam programas de ensino extensivo como cursos, exposições, eventos e/ou palestras, pois contribuem significativamente para a promoção desta ciência no meio escolar. Programas esses que tem sua importância deveras presente na atualidade, uma vez que instituições de ensino costumam contribuir essencialmente para a difusão científica, oportunizando à comunidade alcançar conhecimentos a partir de práticas não-formais de ensino, como também sugerem os autores (Almeida et al., 2013). 
A importância de museus, laboratórios e exposições não foi evidenciada em nenhuma das coleções, no máximo exibindo imagens de fósseis - sem identificação - que continham em alguma instituição. Almeida et al. (2013) promove o pensamento de que ao integrar o conhecimento paleontológico teórico com práticas expositivas, tem-se uma relevante estratégia educacional, reforçando os prejuízos da falta de ocorrência deste assunto em materiais didáticos. Muitos autores fundamentam a importância de estimular o respeito aos espaços de natureza expositiva durante as aulas, por levar o acesso ao tema paleontologia para além dos limites das escolas (Schwanke \& Silva, 2004; Mello et al., 2005).

Além de todas contribuições para/na educação e cultura, a paleontologia também contribui na economia, aspecto evidenciado apenas pela CLDc4 que menciona vários combustíveis fósseis e suas origens. Este assunto, de acordo com Nizer (2019), contribui muito para valores econômicos, pois fornece informações que permitem a extração de vários combustíveis fósseis e suas origens para melhor exploração dos materiais. $\mathrm{O}$ autor ainda indica que o turismo paleontológico, que costuma se tratar de visitações a parques temáticos, museus e sítios, são um potencial tipo efetivo de atrativo econômico (Nizer, 2019).

Como frequentes meios utilizados para comunicação, obtenção de informação e acesso à acultura, as mídias costumam trazer informações equivocadas e que reforçam um senso comum (Novais et al., 2015). Nenhuma das coleções discute a respeito da relação da paleontologia com as mídias, algo que seria interessante de ser promovido junto aos estudantes, afim de desmitificar suas preconcepções sobre informações de senso comum.

Tendo um bom espaço na cultura, no ensino e uma presente contribuição econômica para a humanidade, era de se esperar que assuntos paleontológicos estivessem mais valorizados em materiais didáticos. Sendo o próprio LD essencial na difusão de informações socioculturais, é perceptível o esquecimento das coleções analisadas para com a paleontologia.

\subsubsection{Categoria Didática}

$\mathrm{Na}$ categoria Didática, todas coleções se encontram bem estruturadas, com imagens a respeito de fósseis reais e ilustrações de espécies extintas, entre outros exemplos de "paleo-arte", todas com as devidas legendas e orientações. Foi possível observar que apenas as coleções CLDc1 e CLDc5 exibem imagens de paleontólogos atuando em sítios ou em estudos, conforme exposto no Quadro 14.

Quadro 14: Pontuação de cada coleção analisada referente à Categoria Didática, onde 20 representa a pontuação máxima.

\begin{tabular}{|c|c|c|c|c|c|c|c|}
\hline COLEÇÃO & Q1 & Q2 & Q3 & Q4 & Q5 & Q6 & Total \\
\hline CLDc1 & 4 & 2 & 2 & 4 & 2 & 2 & $\mathbf{1 6}$ \\
\hline CLDc2 & 2 & 2 & 1 & 4 & 4 & 2 & $\mathbf{1 5}$ \\
\hline CLDc3 & 0 & 2 & 1 & 4 & 2 & 2 & $\mathbf{1 1}$ \\
\hline CLDc4 & 4 & 2 & 1 & 4 & 2 & 2 & $\mathbf{1 5}$ \\
\hline CLDc5 & 2 & 2 & 2 & 4 & 2 & 2 & $\mathbf{1 4}$ \\
\hline
\end{tabular}

Fonte: Dados da Pesquisa.

Dentre as estratégias didáticas que devem ser estimuladas para o ensino significativo de paleontologia, a mais relevante é a contextualização. Segundo Pessano et al. (2015), contextualizar aproxima o conhecimento científico dos alunos com muita eficiência, principalmente se tratando de temas que apresentem articulações entre si na aprendizagem integral.

Recursos que possibilitem uma visão mais realista e proximal ao cotidiano dos estudantes, conseguem trazer um contato mais completo e significativo, pois, como ressalta Zucon et al. (2010), a visualização dos processos de coleta de fósseis e do próprio material paleontológico tendem a ajudar na compreensão do estudo dessa temática despertando melhor o interesse na aprendizagem. É importante ressaltar que todos as coleções apresentavam pelo menos uma figura realista ao trabalhar temas 
da paleontologia ou conteúdos relacionados, evidenciando que os LD seguem a vertente de que, no ensino, a visualização direta dos estudantes com os objetos de estudo são essenciais para a capacidade de relacionar o estudo ao meio em que vivem (Zucon \& Silva, 2010).

Atividades e/ou exercícios a respeito de paleontologia se encontram melhor elaboradas nas CLDc1 e CLDc4, abordando assuntos que compõe a temática em questões mais completas. Nas CLDc2 e CLDc5 são mais gerais e em menor quantidade, no máximo se referindo a definições de termos. Por fim, na CLDc3 as atividades a respeito da temática estão ausentes. A importância de sua abordagem vai ao encontro das ideias de Santos e Carneiro (2013), que corroboram indicando que a utilização do LD como ferramenta de caderno de exercícios é uma estratégia coerente na visão linear atual de ensino e aprendizagem para com as escolas.

Contextualização, ilustrações e atividades são características que deveriam estar presentes nos LD e com resultados positivos por conta de o viés dos materiais ser a didática. O potencial da paleontologia para esse objetivo é visível, principalmente identificando a importância de temas interdisciplinares e que proponham a comunicação entre assuntos científicos. É preocupante observar a ausência das características analisadas nesta categoria em uma das coleções, pois é indispensável a presença das mesmas para o ensino de paleontologia.

\subsection{Classificação Final}

Dentre todas as categorias, nenhuma coleção obteve pontuação máxima e consequentemente, nenhuma atingiu a pontuação total final, nem mesmo chegaram a atingir o conceito Ideal. Ainda que nenhum tenha atingido o conceito Insuficiente, as coleções CLDc3 e CLDc5 receberam conceito Básico - Ba e as demais, conceito Regular - Re, conforme o Quadro 15.

Quadro 15: Classificação final das coleções analisadas de acordo com a matriz utilizada.

\begin{tabular}{|c|c|c|c|c|c|c|c|c|}
\hline COLEÇãO & C1 & C2 & C3 & C4 & C5 & C6 & P & Classificação \\
\hline CLDc1 & 6 & 20 & 2 & 11 & 4 & 16 & $\mathbf{5 9}$ & Re \\
\hline CLDc2 & 7 & 19 & 9 & 14 & 3 & 15 & $\mathbf{6 7}$ & $\mathbf{R e}$ \\
\hline CLDc3 & 3 & 21 & 3 & 7 & 4 & 11 & $\mathbf{4 9}$ & Ba \\
\hline CLDc4 & 5 & 24 & 6 & 9 & 6 & 15 & $\mathbf{6 5}$ & $\mathbf{R e}$ \\
\hline CLDc5 & 4 & 12 & 4 & 11 & 3 & 14 & $\mathbf{4 8}$ & $\mathbf{B a}$ \\
\hline
\end{tabular}

Fonte: Autores.

É curioso relembrar que a ordem de numeração das coleções é um "ranking", ressaltando que a CLDc1, por mais que seja a coleção mais selecionada na cidade de Uruguaiana, não ficou entre as duas maiores pontuações na classificação final. Pode-se observar que coleções como a CLDc2 e CLDc4 abordaram de forma mais completa a paleontologia, por mais que todas coleções não expressassem especificamente muitos dos conteúdos relacionados à temática em seus livros. Isso indica que se tratam de conteúdos negligenciados para com o ensino de ciências que, segundo as pontuações finais diagnosticadas, não fornecem os conteúdos necessários para serem desenvolvidos em sala de aula de forma eficiente e significativa.

Portanto, o fato de a paleontologia ser encontrada de forma pouco abrangente, incompleta ou até mesmo ausente, evidencia que os LD pecam neste quesito quando deveriam promover meios mais significativos para o exercício desta temática tão essencial para a compreensão da origem do mundo (Moraes et al., 2007). Sendo assim, por se tratar de um aliado conceitual pedagógico utilizado por docentes de todo o país, os LD necessitam salientar os conhecimentos apresentados a respeito da paleontologia, dada a sua importância para o ensino de ciências e a humanidade. 


\section{Considerações Finais}

Diante dos resultados obtidos, evidenciou-se a desvalorização da temática paleontologia nos LD científicos analisados, devido a superficialidade nas definições de termos científicos frequentes na temática, o que resultou na falta de promoção da articulação entre os conceitos paleontológicos e os demais temas que apresentam interface com as outras áreas das ciências naturais.

Por mais importante e necessária que a temática paleontologia seja para o ensino de ciências, a mesma é representada de forma descontextualizada e com pouca associação com o cotidiano, dificultando assim o processo de aprendizagem dos estudantes. Este fato é preocupante, uma vez que os LD analisados já estão adequados à BNCC, documento normativo atual, que, tal qual os PCN, também negligencia a temática.

A partir deste estudo, observamos a necessidade de os LD legitimarem a temática paleontologia em suas coleções, considerando que é na escola onde os estudantes constroem seus saberes e entendem o funcionamento do mundo que vivem, utilizando o mesmo como aliado deste processo.

Realmente os LD analisados demonstram a existência de um esquecimento dos conteúdos paleontológicos. Porém pensando na modernização, os LD poderiam contemplar mais dados tecnológicos, mais aliados que levem a fontes que tragam mais conteúdo, como o uso de links e QRCode para acesso às informações complementares, e maior reforço da interdisciplinaridade por conta de possíveis modificações na abordagem das temáticas científicas.

Por fim, acreditamos que os resultados deste estudo contribua para o desenvolvimento de pesquisas posteriores que busquem a qualificação do ensino da temática paleontologia, possibilitando meios para a promoção da contextualização e também a articulação entre as diferentes disciplinas curriculares, considerando estas estratégias essenciais e necessárias para o alcance de uma aprendizagem significativa em todos os níveis de ensino.

\section{Referências}

Almeida, L. F., Zucon, M. H., de Souza, J. F., Reis, V. S., \& Vieira, F. S. (2014). Ensino de Paleontologia: uma abordagem não-formal no Laboratório de Paleontologia da Universidade Federal de Sergipe. Terrae Didática, 10(1), 14-21. https://periodicos.sbu.unicamp.br/ojs/index.php/td/article/view/8637384

Anelli, L. E. (2002). O passado em suas mãos: guia para coleção de réplicas. EDUSP.

Aráujo, L. A. L. (2017). Evolução Biológica: da pesquisa ao ensino. Editora Fi.

Brasil, Fundação Nacional de Desenvolvimento da Educação. (2017). Histórico. http://www.fnde.gov.br/programas/programas-do-livro/livrodidatico/historico

Brasil, Ministério da Educação. (2020). Guia de Livros Didáticos - PNLD 2020 - CIÊNCIAS.

Brasil, Secretaria de Educação Fundamental. (1998). Parâmetros curriculares nacionais: Ciências Naturais. Secretária de Educação Fundamental. Brasília: $\mathrm{MEC} / \mathrm{SEF}$.

Bueno, R. \& Macedo, T. (2018). Inspire Ciências. FTD.

Carnevalle, M. R. (2018). Projeto Araribá Mais - Ciências. Moderna.

Carvalho, I. D. S. (2010). Paleontologia: Conceitos e Métodos. Editora Interciência.

Castro, L. R. B.; De Carvalho, A. V.; Soares, J. R. \& Castro Pessano, E. F. (2019). Os biomas brasileiros nos livros didáticos de ciências: um olhar ao pampa gaúcho. Revista Electrónica de Investigación en Educación en Ciencias, 14(1), 38-49.

Cavalcante, M. S. D.; Pinho, M. J. \& Andrade, K. S. (2015). Interdisciplinaridade e livro didático: interfaces (im)possíveis? Revista do GELNE, 17(1/2), 213234.

Compiani, M. (2021). Comparações entre a BNCC atual e a versão da consulta ampla, item ciências da natureza. Ciências Em Foco, 11(1), 16. https://econtents.bc.unicamp.br/inpec/index.php/cef/article/view/15027

Delizoicov, D.; Angotti, J. A. \& Pernambuco, M. M. C. A. (2002). Ensino de Ciências: fundamentos e métodos. Cortez.

Dos Reis, M. A. F., Carvalho, C. V. A., Carvalho, J. V., Rodrigues, M. A. C., Medeiros, M. A. M., Villena, H. H., Oliveira, F. M., \& Dornelas, V. R. (2005). Sistema multimídia educacional para o ensino de geociências: uma estratégia atual para a divulgação da paleontologia no ensino fundamental e médio. Anuário do Instituto de Geociências, 28(1), 70-79. 
Duarte, S. G.; Arai, M., Passos, N. Z. G. \& Dolores, M. W. (2016). Paleontologia no ensino básico das escolas da rede estadual do Rio de Janeiro: uma avaliação crítica. Anuário do Instituto de Geociências da UFRJ, 39(2), 124-132.

Franco, M. L. P. B. (1992). O livro didático e o Estado. ANDE, 1(5), 19-24.

Frison, M. D., Vianna, J., Chaves, J. M., \& Bernardi, F. N. (2009). Livro didático como instrumento de apoio para construção de propostas de ensino de ciências naturais. Encontro Nacional de Pesquisa em Educação em Ciências, 7, 1-13.

Ghilardi, R. P., Ribeiro, R. N. S., \& Elias, F. A. (2007). Paleodesign: uma nova proposta metodológica e terminológica aplicada à reconstituição em vida de espécies fósseis. Paleontologia: Cenários de Vida, 2, 61-70.

Gil, A. C. (2019). Métodos e técnicas de pesquisa social (7a ed.). Atlas.

Gewandsnajder, F. \& Pacca, P. (2018). ProjetoTelaris: Ciências. Ática.

Godoy, L. (2018). Ciências: Vida e Universo. São Paulo: FTD.

Heirich, C. M., Matsumura, W. M. K., Myszyski-Junior, L. J., Serdoko, D. \& Bosseti, E. P. O. (2015). O aprendizado da Paleontologia no Ensino Básico da cidade de Tibagi-PR. In Paleo PR/SC, Paraná, Brasil. http://www.fecilcam.br/paleoprsc/data/uploads/o-aprendizado-da-paleontologia-no-ensino-basico-dacidade-de-tibagi-n-pr.pdf

Izaguirry, B. B. D., Ziemann, D. R., Muller, R. T., Dockhorn, J., Pivotto, O. L., Costa, F. M., Alves, B. S., Ilha, A. L. R., Ramos, A. L., Stefenon, V. M., \& Dias-da-Silva, S. (2013). A paleontologia na escola: uma proposta lúdica e pedagógica em escolas do município de São Gabriel, RS. Cadernos da Pedagogia, 7(13), 2-16.

Jordão, T. C. (2010). Recursos digitais de aprendizagem. Revista Tecnologias na Educação 1(1).

Kato, D. S., \& Kawasaki, C. S. (2011). As concepções de contextualização do ensino em documentos curriculares oficiais e de professores de ciências. Ciência \& Educação, 17(1), 35-50.

Leite, G. L. D., \& De Sá, V. G. M. (2010). Apostila: Taxonomia, Nomenclatura e Identificação de Espécies. Belo Horizonte: Universidade Federal de Minas Gerais.

Manzig, P. (2015). Divulgação científica: a paleontologia nos museus brasileiros. Ciência e Cultura, 67(4), 54-55.

Martello, A. R., Novais, T., Oleques, L. C., Leal L. A. \& Rosa, Á. A. S. (2015). A inserção da paleontologia no ensino fundamental em diferentes regiões do Brasil. TerraDidatica, 11(1), 33-41. http://www.ige.unicamp.br/terraedidatica

Mello, F. T., Mello, L. H. C., \& Torello, M. B. F. (2005). A Paleontologia na Educação Infantil: alfabetizando e construindo o conhecimento. Ciência e Educação, 11(3), 397-410.

Moraes, S. S., Santos, J. F. S. \& Brito, M. M. M. (2007). Importância dada à paleontologia na educação brasileira: Uma análise dos PCN e dos livros didáticos utilizados nos colégios públicos de Salvador, Bahia. In I. S. Carvalho et al. (eds.). Paleontologia: Cenários de Vida. (pp. 71-75). Rio de Janeiro: Editora Interciência.

Neto, V. D. P. \& Paesi, R. A. (2017). Sistemática filogenética: Abordando a evolução em sala de aula. In L. A. L. Araújo. Evolução Biológica: Da pesquisa ao ensino. (pp. 245-262). Porto Alegre: Editora Fi.

Novais, T., Martello, A. R., Oleques, L. C., Leal, L. A. \& Da-Rosa, A. A. S. (2015). Uma experiência de inserção da paleontologia no ensino fundamental em diferentes regiões do Brasil. Terra Didatica, 11(1), 33-41. https://www.ige.unicamp.br/terraedidatica/v11_1/PDF11-1/111-\%204-105.pdf

Núñez, I. B., Ramalho, B. L., Silva, I. K. P. \& Campos, A. P. N. (2003). A seleção dos livros didáticos: um saber necessário ao professor. O caso do ensino de Ciências. Revista Iberoamericana de Educación, 33(1), 1-11.

Oliveira, J. P. T. (2014). A eficiência e/ou ineficiência do livro didático no processo de ensino-aprendizagem. In IV congresso ibero-americano de política e administração da educação/ VII congresso Luso-Brasileiro de política e administração da educação. Anpae. https://anpae.org.br/IBERO_AMERICANO_IV/GT4/GT4_Comunicacao/JoaoPauloTeixeiradeOliveira_GT4_integral.pdf

Pessano, E. F. C., Dávila, E. S., Ocampo, D. M., Miralha, C. T. T., Folmer, V. \& Puntel, R. L. (2015). O rio uruguai como estratégia de contextualização para ensino em uma unidade de restrição de liberdade para adolescentes. Góndola, Enseñanza Y Aprendizaje De Las Ciencias, 10(1), 74-101.

Romanatto, M. C. (2004). O livro didático: alcances e limites. Encontro paulista de matemática, 7, 1-11. http://www.miltonborba.org/CD/Interdisciplinaridade/Anais_VII_EPEM/mesas_redondas/mr19-Mauro.doc

Santos, W. L. P. \& Carneiro, M. H. S. (2013). Livro Didático de Ciências: Fonte de Informação ou Apostila de Exercícios?. Revista Contexto \& Educação, 21(76), 201-222. DOI: 10.21527/2179-1309.2006.76.201-222. https://revistas.unijui.edu.br/index.php/contextoeducacao/article/view/1103

Schwanke, C. \& Silva, M. A. J. (2004). Educação e Paleontologia. In I. S. Carvalho. (2007). Paleontologia: cenários da vida. (pp. 123-130). Rio de Janeiro: Interciência.

Silva, A. C., \& Junior, N. M. (2017). Análise do conteúdo de fungos nos livros didáticos de biologia do ensino médio. Revista Ciências \& Ideias, 7(3), 235273.

Sousa, R. C., Da Silva, I. R., Dos Santos, S. F., De Figueiredo, A. E. Q. \& Fortier, D. C. (2016). O ensino de Paleontologia nas escolas públicas estaduais de Floriano-PI na concepção dos docentes. In III Congresso Nacional de Educação no Estado do Rio Grande do Norte. (2018). Anais... Rio Grande do Norte. http://www.editorarealize.com.br/revistas/conedu/trabalhos/TRABALHO_EV056_MD4_SA18_ID10955_17082016193329.pdf 
Research, Society and Development, v. 10, n. 7, e40610716641, 2021

(CC BY 4.0) | ISSN 2525-3409 | DOI: http://dx.doi.org/10.33448/rsd-v10i7.16641

Thompson, M. \& Rios, E. P. (2018). Observatório de Ciências. Moderna.

Vieira, F. S., Zucon, M. H. \& Santana, W. S. (2010). Análise dos conteúdos de paleontologia nos livros didáticos de biologia e nas provas de vestibular da UFS e do ENEM. EDUCON Colóq. Intern. Educação e Contemporaneidade. 4, 1-10.

Zamberlan, E. S. J \& Silva, M. R. (2009). O evolucionismo como princípio organizador da biologia. Temas \& Matizes, 8(15), 27-41.

Zucon, M. H. \& Silva, M. A. (2010). Violência Cultural e os Conhecimentos Paleontológicos de Sergipe. In: R. R. Jalaliv. (2010). Ensino de Paleontologia: uma abordagem não formal no Laboratório de Paleontologia da Universide. (pp. 283-300). Criação.

Zucon, M. H., Vieira, F. S., Prazeres, M. F. F. \& Dantas, M. A. T. (2010). O ensino de Paleontologia e a percepção dos alunos do curso de Biologia da Universidade Federal de Sergipe. In Anais do IV Colóquio Intern. Educ. e Contemporaneidade. EdUFS. http://educonse.com.br/2010/eixo_05/E5-41.pdf 